\title{
Performance of the AMS02 Electromagnetic Calorimeter in Space
}

\author{
Marco Incagli ${ }^{* \dagger}$ \\ Istituto Nazionale di Fisica Nucleare - Pisa \\ E-mail: marco.incagli@pi.infn.it
}

The Alpha Magnetic Spectrometer (AMS-02) is a high-energy particle detector deployed on the International Space Station (ISS) since May 19, 2011 to conduct a long-duration mission on fundamental physics research in space. The main scientific goals of the mission are the detection of antimatter and dark matter through the study of the spectra and fluxes of protons, electrons, nuclei until the iron, their antiparticles, and gamma-rays in the $\mathrm{GeV}$ to $\mathrm{TeV}$ energy range. The Electromagnetic CALorimeter (ECAL) is required to measure electron, positron and gamma spectra and to discriminate electromagnetic showers from hadronic cascades. To fulfill these requirements the ECAL is based on a lead/scintillating fiber sandwich, for a total of 17 radiation lengths for perpendicular incidence, providing a 3 Dimensional imaging reconstruction of the showers. The high granularity consists of 18 samplings in the longitudinal direction, and 72 samplings in the lateral direction. Measurements of ECAL parameters in space and performance in term of energy and angular resolutions, linearity and proton rejection will be reviewed.

Technology and Instrumentation in Particle Physics 2014,

2-6 June, 2014

Amsterdam, the Netherlands

\footnotetext{
* Speaker.

†n behalf of the AMS02 collaboration
} 


\section{Calorimeter}

The AMS-02 ECAL ${ }^{1}$ consists of a lead/scintillating fiber sandwich with an active area of $648 \times 648 \mathrm{~mm}^{2}$ and a thickness of $166 \mathrm{~mm}$. The calorimeter is composed of superlayers, each $18.5 \mathrm{~mm}$ thick and made of 11 grooved $1 \mathrm{~mm}$ thin lead foils interleaved with layers of $1 \mathrm{~mm}$ diameter scintillating fibers glued to the foils by means of optical epoxy. The resulting composite structure has a relative lead-fiber-glue volume composition of 1:0.57:0.15 and an average density of $6.8 \pm 0.2 \mathrm{~g} / \mathrm{cm}^{3}$ (see fig.1).

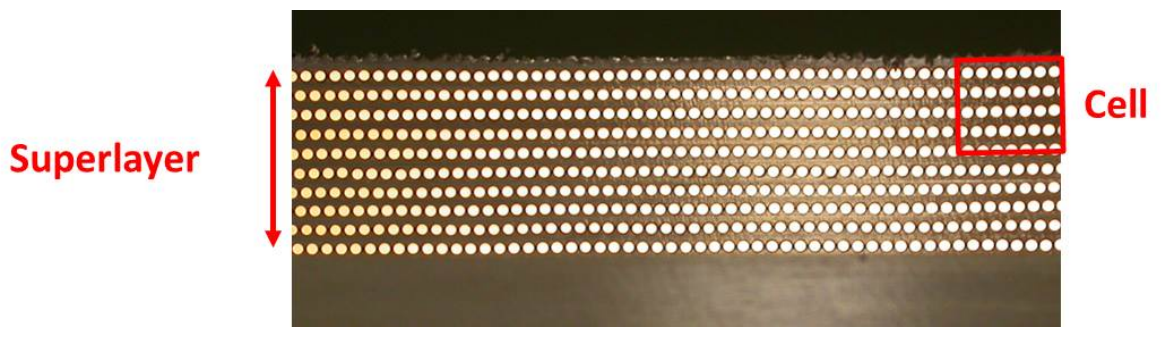

Figure 1: ECAL structure and cell dimensions. Each fiber has a diameter of $1 \mathrm{~mm}$.

In each superlayer, fibers run in one direction only. The detector imaging capability is obtained by stacking superlayers with fibers alternatively parallel to the x-axis (5 layers) and y-axis (4 layers). In AMS, the dipole magnetic field is oriented along the $\mathrm{x}$-axis. The active part of the calorimeter, also called pancake, has a weight of $496 \mathrm{~kg}$, for a total weight of $638 \mathrm{~kg}$, including mechanical structure and readout cables. Its thickness corresponds to about 17 radiation lengths.

Fibers are read out at one end only by four anode Hamamatsu R7600-00-M4 photomultipliers (PMTs); each anode covers an active area of $9 \times 9 \mathrm{~mm}^{2}$, corresponding to 35 fibers, defined as a cell. In total ECAL is subdivided into 1296 cells (324 PMTs) and this allows for a sampling of the longitudinal shower profile by 18 independent measurements.

\section{Mechanical assembly}

The ECAL mechanical assembly supports the calorimeter, the PMTs and the attached electronics. It is designed to minimize the weight while ensuring a first resonance frequency above 50 $\mathrm{Hz}$, the capability to withstand accelerations up to $12 \mathrm{~g}$ in any direction and to limit temperature gradients at the Front End Electronics (FEE) level to less than one degree during each orbit.

The optimiziation of the mechanical structure through finite element studies led to an aluminum alloy support frame composed of four lateral panels, which contain the PMTs, and to top and bottom honeycomb planes.

\section{Light collection}

Each superlayer is read out by 36 PMTs, arranged alternatively on the two opposite ends in order to read out each fiber with no dead areas. As shown in fig.2, each PMT is surrounded by a magnetic shield which also contains the light guides, the PMT base and FEE. 
To maximize light yield and to reduce cross-talk between cells, photons from the fibers are collected by mean of individual Plexiglas light guides. Silicon optical joints are positioned on both ends of light guides, to ensure a good optical transmission from the fibers to the PMTs, even after high mechanical stresses are applied.

To reduce the residual magnetic field below $20 \mathrm{G}$, PMTs are positioned inside $1 \mathrm{~mm}$ thick soft iron square tubes $30.5 \mathrm{~mm}$ wide and $74 \mathrm{~mm}$ long.

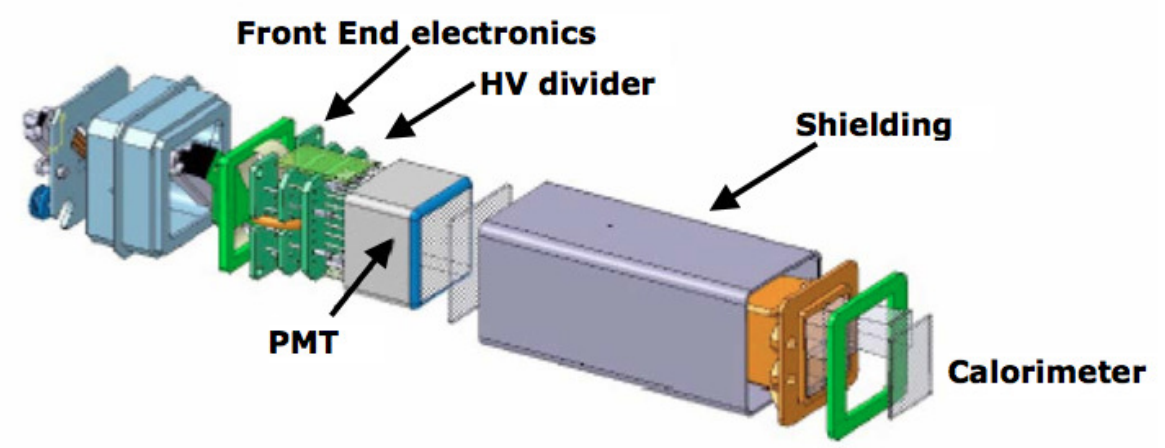

Figure 2: Scheme of the PMT light collection system.

\section{Electronics}

In addition to the FEE mounted directly on the calorimeter, ECAL is instrumented with several custom developed electronics subsystems for data reduction, slow control, trigger and high and low voltage. Each board is composed of two completely independent sectors that are separately powered and capable of the same functionality. In case of any fault, it is possible to switch from one sector to the other, so to maintain full functionality of ECAL.

\subsection{Front End Electronics (FEE)}

For each cell the readout system is required to process signals with a good linearity over a wide dynamic range, from a single minimum ionizing particle, which deposits about $7 \mathrm{MeV}$ per cell, up to about $60 \mathrm{GeV}$, the maximum energy expected in a single cell by the electromagnetic shower of $1 \mathrm{TeV}$ electrons. To fulfil this requirement, each anode signal is split into two equal parts by voltage dividers and the two parts are amplified in two electronics channels with a gain ratio of about 33 (see fig.7). The two channels are called High Gain (HG) and Low Gain (LG), respectively. The ratio between these two gains, called High Gain Low Gain ratio, or HGLG, is a fundamental parameter that provides the connection between the particles used for equalization, the Minimum Ionizing Particles, and the high energy electron/positron showers. The HGLG has to be constantly monitored with flight data; this procedure will be described in section 6 .

The digitized outputs from 9 PMTs are sent via an intermediate board (EIB) mounted on ECAL mechanical support and then to the data reduction boards $(E D R)$, hosted in the 2 ECAL 
readout crates (ECRATE) located on the diagonal beams of the AMS support structure. The comparator outputs from the last dynode are sent to the trigger boards (ETRG), which are also hosted in the ECRATEs.

\subsection{DAQ system}

ECAL readout follows the overall AMS-02 design. The EDR receives the digitized signals from 27 PMTs (3 EIB) over Low Voltage Differential Signaling (LVDS) lines, subtracts pedestals, suppresses zeros and sends the results throughout the backplane to the next node in the DAQ chain, the $J I N F$. The EDR also acts as a bridge for the FEE power supply (+3.5, -2.5 VDC) and for the control of EIBs using single ended TTL (5 V) signaling.

A description of ECAL boards can be found in $\left({ }^{1}\right)$.

\section{ECAL standalone gamma trigger}

The excellent imaging capabilities of ECAL allows for the implementation of a very efficient standalone trigger for photons with energies down to $2 \mathrm{GeV}$.

The trigger is made in two steps: a fast decision, available within $180 \mathrm{~ns}$, provided by the count of PMTs above threshold in the 6 central superlayers of the calorimeter and a Level 1 trigger decision, available before $1 \mu \mathrm{s}$, obtained with a fast reconstruction of particle direction. The photon direction is calculated by using the centers of gravity of the three superlayers with parallel fibers both in $\mathrm{x}$-view and in y-view. Particles with an inclination larger than 20 degrees are rejected, ensuring that the trajectory passed through the full AMS acceptance.

The trigger efficiency can be evaluated by using the events tagged by the prescaled "unbiased trigger". The result for electrons is shown in fig.3.

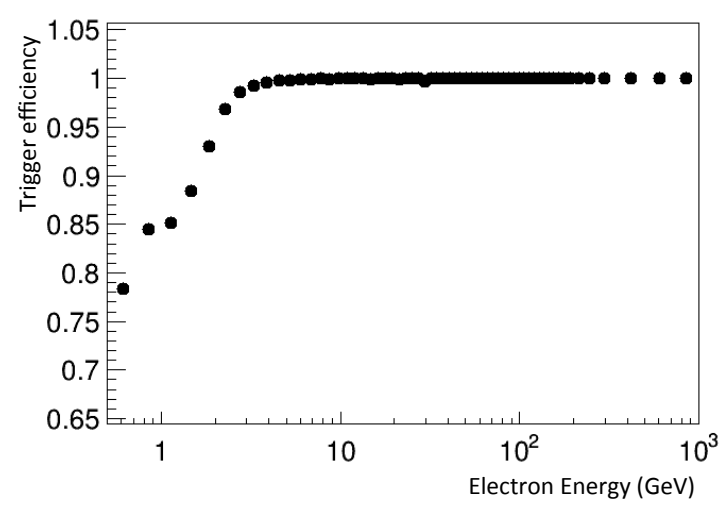

Figure 3: Trigger efficiency for electrons/positrons as derived from the prescaled unbiased trigger.

In order to remove events which enter in AMS02 from the side, a set of scintillator counters is placed inside the magnet. These counters, named AntiCoincidence Counters (ACC), are used as veto in the trigger logic. However, due to the backsplash of electrons impacting on ECAL, the 


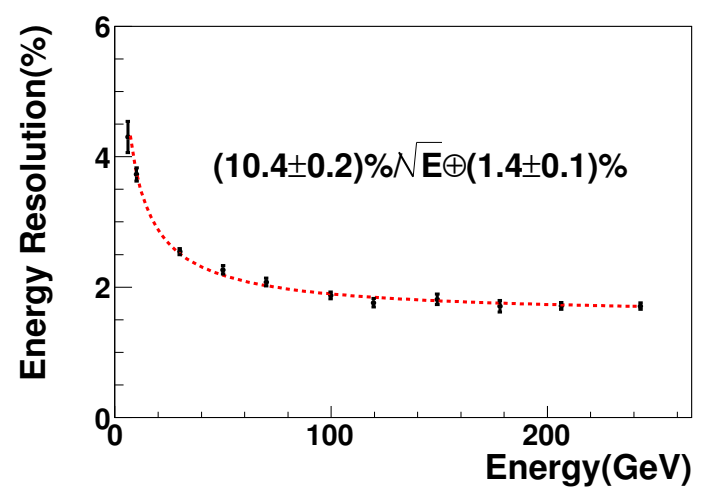

Figure 4: ECAL energy resolution from Test Beam data.

ACC can be trigger by genuine events. For this reason, the ECAL trigger is used also to inhibit the ACC veto, enhancing the charged trigger performance on high energy electrons.

\section{ECAL performance}

In July 2006 and in October 2007, the ECAL flight model was exposed to the CERN SPS beam line $\mathrm{H} 4$ with hadrons and electrons of energies in the range 6 to $250 \mathrm{GeV}$. In August 2010, after the integration of ECAL with the rest of the apparatus, the full AMS02 detector was tested in the beam line $\mathrm{H} 8$ with hadrons and electrons of energies 10 to $180 \mathrm{GeV}$ and with the primary proton beam line of $400 \mathrm{GeV}$.

During these tests, the procedures for calorimeter equalization and calibration have been verified and ECAL performance have been measured. The ECAL energy resolution for normal incidence tracks is shown in fig.4 and it can be parametrized as $\sigma(E) / E=(10.4 \pm 0.2) \% / \sqrt{E(\mathrm{GeV})} \oplus$ $(1.4 \pm 0.1) \%$. Since the AMS-02 angular acceptance is limited to $\simeq 20^{\circ}$, this resolution doesn't change appreciably for inclined tracks.

The reconstruction of the shower energy is performed after a set of equalization and calibration procedures have been applied at cell level. In order to guarantee the stability of the apparatus response in flight, it is necessary to study the dependence of these corrections with time, temperature and, in general, ambient conditions. This will be described in the next section.

\section{Stability of the ECAL response versus time and temperature}

Before clustering the hits, the following steps need to be performed (a more detailed description can be found in ${ }^{1}$ ):

- correction for fiber length attenuation;

- equalization of all the cells using MIPs;

- calibration of the ratio HighGain/LowGain. 


\subsection{Stability of fiber length attenuation}

The signal amplitude at a distance $x$ from the PMT face is attenuated according to a function which is normally parametrized in terms of two lengths (the "fast attenuation", $\lambda_{f}$, and the "slow attenuation", $\lambda_{s}$ ):

$$
A(x)=f e^{\left(-x / \lambda_{f}\right)}+(1-f) e^{\left(-x / \lambda_{s}\right)}
$$

where $f$ is the weight of the fast attenuation process. For AMS-02 fibers typical values for these parameters are: $f=0.17, \lambda_{f}=110 \mathrm{~mm}, \lambda_{f}=2605 \mathrm{~mm}$.

The attenuation length parameters of each cell have been measured on ground with muon cosmic rays. These parameters are regularly checked in order to anticipate an eventual yellowing of the fibers. After three years of data taking, the fiber response is stable.

\subsection{Temperature effects on cell equalization}

The cell response is equalized by using the protons which behave as MIPs. A typical signal of a MIP is shown in fig.5; the distribution is fitted with a Landau curve convoluted with a gaussian and the Landau peak is taken as reference value for equalization.

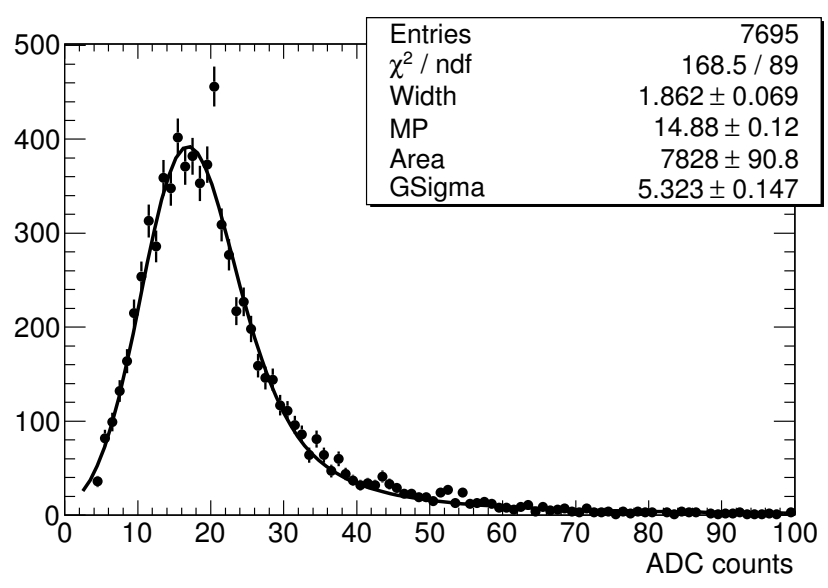

Figure 5: Typical distribution of MIP signal in an ECAL cell.

A dependence of the PMT gain from temperature is expected. This reflects in a shift of the MIP peak, and therefore of the energy scale. To monitor this effect, each ECAL face is equipped with 6 redundant pairs of thermal sensors connected to the aluminum PMT holder structure, 2 pairs on each side and 2 pairs on the face center. The temperature of a given PMT is determined by the closest pair of sensors ${ }^{1}$. Fig. 6 shows the correlation between the PMT temperature and the MIP peak position. An average slope of $0.25 \% /{ }^{\circ} \mathrm{C}$ in the energy scale is observed, which is corrected during offline reconstruction.

\footnotetext{
${ }^{1}$ We have performed also an interpolation algorithm, to have a more precise determination of each specific PMT temperature, but no appreciable difference in the results has been observed.
} 


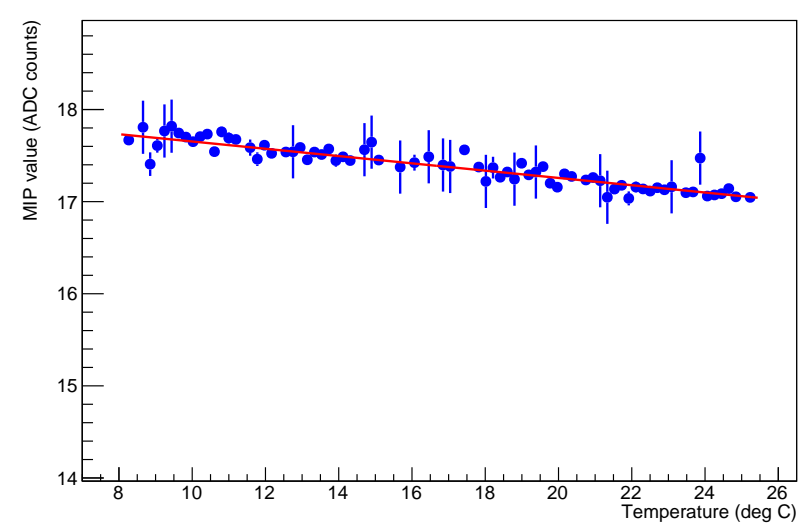

Figure 6: Position of the MIP peak, or Most Probable Value (MPV) as a function of temperature. An Energy Scale variation of $0.25 \% /{ }^{\circ} \mathrm{C}$ is observed.

\subsection{Temperature effects on cell HighGain/LowGain}

As already described in section 4.1, in order to extend the dynamical range of the ECAL readout, the signal of each channel is split in two paths with different gains. The ratio of the two amplifications $(H G L G)$ is measured for each cell and its stability as a function of the temperature monitored.

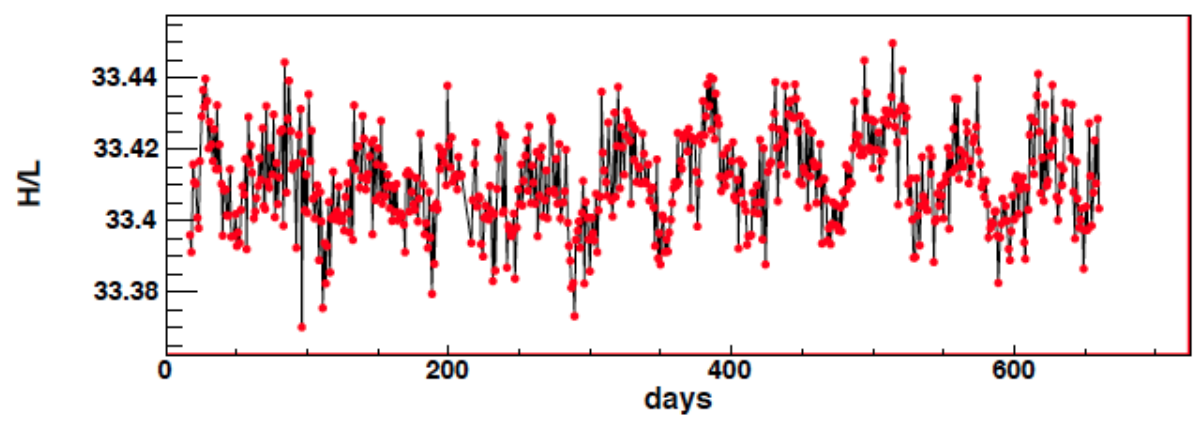

Figure 7: Ratio High Gain/Low Gain $(H / L)$ as a function of time (days from beginning of data taking). A stability at the per mill level is observed.

Being a ratio between two different amplifications, its dependence with the temperature is expected to be less pronounced than the one of the MIP peak. This is confirmed in fig.7. The average fluctuation of $H G L G$ in the first two years of data taking is at the one per mill level.

As a consequence, no correction is applied in reconstruction.

\subsection{Dependence from other ambient variables}

After temperature corrections have been applied, as described in the previous section, for a limited number of PMTs futher effects are observed in the MIP peak position. 
An example of these residual effects are shown in fig.8 for two PMTs. In 8.left, the response of the 4 cells of a specific PMT, after temperature corrections, is shown as a function of time. During the first three months of data taking, a slow drift of $25-30 \%$ is observed. Presumably, this is due to a slow outgassing of the material surrounding the PMT, or by the PMT itself, resulting in a gain variation. In 8.right, the response of a PMT to MIP particles is compared with the adjacent ones. The PMT under observation manifests a long term drift.
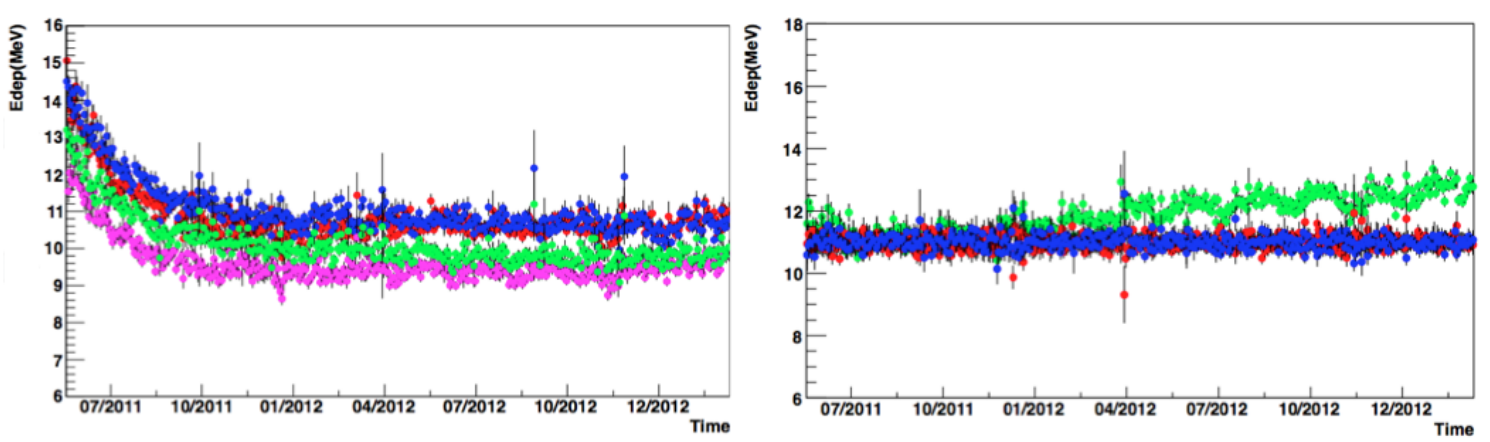

Figure 8: Left: response of the 4 cells of a specific PMT showing the long time taken to stabilize the response after installation on the International Space Station. Right: response of a PMT vs time (green), compared with the two adjacent ones, showing long term drift effects. These effects are corrected by daily equalization, as described in the text.

In order to keep all these effects under control, the MIP equalization is performed daily and the PMT energy response corrected accordingly. After this additional correction, the energy scale of all cells is stable at the \% level.

\subsection{Angular resolution}

The angular resolution for electromagnetic showers can be determined with data by comparing the ECAL axis with the Tracker track extrapolation.

The particle direction in ECAL is obtained by fitting a straight line, separately on the $x y$ and $y z$ views, to the Center Of Gravity (COG) in each layer.

The COG is obtained by fitting the profile of the energy deposited in each layer with the shape expected by Montecarlo.

The angular resolution, defined by the 3D angular opening with respect to the tracker direction that contains $68 \%$ of the events, is shown in fig.9; it's better than $1^{\circ}$ at $30 \mathrm{GeV}$ and it reaches a plateau at $0.5^{\circ}$ above energies of $100 \mathrm{GeV}$.

This angular resolution, unusual for a calorimeter, together with the excellent ECAL energy resolution, will allow to investigate the presence of structures in the diffuse photon flux.

\section{Conclusions}

After construction, the Flight Model of the AMS-02 ECAL met successfully the Space Qualification requirements and was tested in a Test Beam facility at CERN in October 2006 and November 


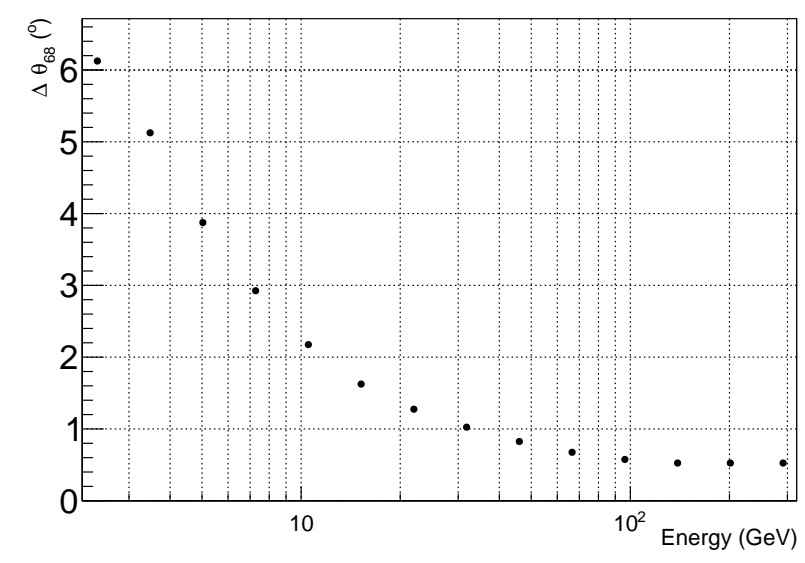

Figure 9: ECAL angular resolution obtained comparing the electron track direction from the tracker with the shower axis (68\% containement angle).

2007. In August 2010, after integration with the rest of the apparatus, the calorimeter was tested again at the CERN Test Beam facility, with the complete AMS-02 detector working.

The ECAL performance measured during different Test Beam studies resulted fully consistent with design specifications.

After almost three years of data taking, ECAL shows no signs of degradation. The energy response has dependends on external conditions, in particular on temperature. This (small) dependence is taken into account during offline reconstruction. The performance measured at Test Beam has been confirmed in flight.

\section{References}

[1] C. Adloff et al., Nucl. Inst. and Methods A, Volume 714,(2013), p. 147-154.

[2] E. Choumilov et al., Nucl. Instr. and Methods A 426 (1999) 625.

[3] R. Hermel, $5^{\text {th }}$ International Meeting on Front-End Electronics (2003). 